\title{
Considering genetic characteristics in German Holstein breeding programs
}

\author{
D. Segelke, ${ }^{* 1}$ H. Täubert, ${ }^{*}$ F. Reinhardt, ${ }^{*}$ and G. Thaller† \\ *Vereinigte Informationssysteme Tierhaltung w.V. (vit), Heideweg 1, 27283 Verden, Germany \\ †Institute of Animal Breeding and Husbandry, Christian-Albrechts-University, 24098 Kiel, Germany
}

\begin{abstract}
Recently, several research groups have demonstrated that several haplotypes may cause embryonic loss in the homozygous state. Up to now, carriers of genetic disorders were often excluded from mating, resulting in a decrease of genetic gain and a reduced number of sires available for the breeding program. Ongoing research is very likely to identify additional genetic defects causing embryonic loss and calf mortality by genotyping a large proportion of the female cattle population and sequencing key ancestors. Hence, a clear demand is present to develop a method combining selection against recessive defects (e.g., Holstein haplotypes HH1-HH5) with selection for economically beneficial traits (e.g., polled) for mating decisions. Our proposed method is a genetic index that accounts for the allele frequencies in the population and the economic value of the genetic characteristic without excluding carriers from breeding schemes. Fertility phenotypes from routine genetic evaluations were used to determine the economic value per embryo lost. Previous research has shown that embryo loss caused by HH1 and HH2 occurs later than the loss for HH3, HH4, and HH5. Therefore, an economic value of $€ 97$ was used against HH1 and HH2 and $€ 70$ against HH3, HH4, and HH5. For polled, €7 per polled calf was considered. Minor allele frequencies of the defects ranged between 0.8 and $3.3 \%$. The polled allele has a frequency of $4.1 \%$ in the German Holstein population. A genomic breeding program was simulated to study the effect of changing the selection criteria from assortative mating based on breeding values to selecting the females using the genetic index. Selection for a genetic index on the female path is a useful method to control the allele frequencies by reducing undesirable alleles and simultaneously increasing economical beneficial characteristics maintaining most of the genetic gain in production and functional traits. Additionally, we applied the genetic index to real data and found a
\end{abstract}

Received April 29, 2015.

Accepted September 27, 2015.

${ }^{1}$ Corresponding author: dierck.segelke@vit.de decrease of the genetic trend for the birth years 1990 to 2006. Since 2010 the genetic index has increased due to a strong increase in the polled frequency. However, further investigation is needed to better understand the biology to determine the correct time of embryo loss and the economic value of fertility disorders.

Key words: genetic index, lethal recessive, genomic evaluation, dairy cattle

\section{INTRODUCTION}

Routine genotyping of a large proportion of the dairy population for genomic evaluation and sequencing of key ancestors provide the opportunity to discover and monitor recessive genetic disorders. VanRaden et al. (2011a) was one of the first research groups who used the genotype pool from routine genomic evaluation to screen for recessive fertility haplotypes. They showed that 3 different Holstein haplotypes (HH1, HH2, and HH3) may cause embryonic loss in the homozygote state. Studies from Denmark (Sahana et al., 2013), the United States (Cooper et al., 2013), and France (Fritz et al., 2013) confirmed these results and identified additional haplotypes (among others, HH4 and HH5). These were also associated with a decrease of fertility due to a potential embryonic loss. Researchers have already found the causal mutation for HH1 (Adams et al., 2012), HH3 (Daetwyler et al., 2014; McClure et al., 2014), and HH4 (Fritz et al., 2013) by using whole genome re-sequencing data of the populations key ancestors (Jansen et al., 2013). However, the causal mutation for HH2 and HH5 is still unknown (McClure et al., 2014). Since the introduction of genomic selection, average inbreeding level increases more than linearly. This increases the risk of potentially many new genetic disorders. In general, for mating decisions and publication, a clear demand is present to combine this potentially large number of disorders with economically beneficial genetic characteristics. Detection and management of genetic disorders will have an effect on genetic gain for fertility traits, animal welfare, and the overall image of the breed (Egger-Danner et al., 2014). Furthermore, economically beneficial traits such as polled (Medugorac et al., 2012; Rothammer et al., 
2014) should be expanded in the dairy populations to avoid dehorning young calves.

In the past, allele frequencies of genetic disorders such as complex vertebral malformation (Agerholm et al., 2001), bovine leukocyte adhesion deficiency (BLAD; Shuster et al., 1992), deficiency of uridine monophosphate synthase (DUMPS; Shanks et al., 1984), and brachyspina (Agerholm et al., 2006; Charlier et al., 2012) were drastically decreased by excluding carrier bulls from AI. Superior bulls were excluded from mating irrespective of their genetic merit and the frequency of the genetic disorder in the population. This results in lower genetic gain for production and functional traits (Van Eenennaam and Kinghorn, 2014). An example by VanRaden et al. (2014) showed that the famous key ancestor Pawnee Farm Arlinda Chief carries the HH1 haplotype. This sire contributed $14 \%$ of his gene variants to the current Holstein population, which increased the milk yield by $\$ 25$ billion. In contrast the costs for mid-term abortions due to HH1 were only $\$ 0.4$ billion.

For each genetic characteristic, comprehensive investigation including allele frequencies, inheritance, economic value, and causal mutation are needed to find the best way of handling the genetic characteristics in breeding programs. In most cases, the carrier frequency can be managed by finding the appropriate mating partner (e.g., mating a carrier sire only to noncarrier dams).

The aim of this study was to derive the economic values for the most important genetic characteristics segregating in German Holsteins and to develop an index of genetic properties summarizing the genetic characteristics and their economic values.

\section{MATERIALS AND METHODS}

\section{Identification of the Considered Genetic Characteristics}

In this study, we used the information of 5 recessively inherited disorders of fertility (HH1 to HH5) and one economically beneficial trait (polled) segregating in the German Holstein population. The characteristics
BLAD and DUMPS were not considered because the carrier frequencies are very low in the current German Holstein population (BLAD $0.2 \%$; DUMPS $<0.1 \%$ ). This means that the probability of mating 2 carriers is very low. The disorders complex vertebral malformation and brachyspina could not be considered based on their patent protection (Georges et al., 2010; Bendixen et al., 2014).

To investigate the development of the carrier frequencies over time, all Holstein animals $(143,511)$ from routine German Holstein genomic evaluation (February 2015) were chosen. The genotype states for HH1 to HH5 were derived by the haplotype based missing homozygosity approach from VanRaden et al. (2011a). Haplotypes were derived using the Beagle (Browning and Browning, 2007) software package. Table 1 shows the used location and the mean minor allele frequency for the birth years 2012 to 2014. Since the end of 2014, the custom Illumina chip (EuroGenomics10KV4) is used for routine genotyping. This chip contains the known causal mutation for $\mathrm{HH} 1, \mathrm{HH} 3$, and $\mathrm{HH} 4$, facilitating the validation of the genotyped status with the derived haplotype status for 7,032 animals. Each causal mutation was added twice to the custom chip. This enables a comparison between the 2 genotype calls and allows to estimate the reproducibility for the identical genome position. The polled state was derived by a method described by Segelke et al. (2013). Briefly, this approach uses for the imputation reference population the polled entries from the herdbook as an additional marker within the polled region (Table 1). For animals with unknown polled state, the additional marker is set to "missing" and afterward imputed using the Beagle (Browning and Browning, 2007) software package. Segelke et al. (2013) showed that the allele error rate for the imputation of the polled state compared with the herdbook entry was $0.2 \%$.

\section{Effects of the Haplotypes on Fertility and Definition of the Economic Values}

The economic value of the fertility defects were indirectly quantified by analyzing the fertility and calving

Table 1. Location and allele frequency of the considered characteristics

\begin{tabular}{lllc}
\hline Characteristic & Chromosome & Map location (bp) & $\begin{array}{c}\text { Mean minor allele frequency }(\%), \\
\text { year of birth 2012-2014 }\end{array}$ \\
\hline HH1 & 5 & $62,394,447$ to $63,983,082$ & 0.88 \\
HH2 & 1 & $93,172,083$ to $98,133,752$ & 0.94 \\
HH3 & 8 & $95,003,606$ to $96,266,647$ & 3.29 \\
HH4 & 1 & $2,128,924$ to $2,942,947$ & 1.26 \\
HH5 & 9 & $92,350,052$ to $93,910,957$ & 2.76 \\
Polled & 1 & 845,494 to $4,052,161$ & 4.06 \\
\hline
\end{tabular}


records from routine genetic evaluation (April 2015) for German Holsteins (Vit, 2015). Nonreturn rate (NRR) 56 and 90 as a measure of increased calving interval was analyzed to estimate the time point of embryonic loss. The NRR is defined as specified period where no consecutive insemination is recorded assuming that the first insemination was successful. If a cow was culled within the lactation, the insemination records of the actual lactation were ignored. The final data set contains 12,439,144 insemination records. For all these records, sire and maternal grandsire of the embryo were genotyped. This means that a carrier status for each genetic characteristic could be derived. Cows and heifers were born between 2000 and 2010. Mean NRR56 and NRR90 heifers were 57.5 and $48.5 \%$ and 52.5 and $42.7 \%$ for cows, respectively. The phenotypic correlation between NRR56 and NRR90 was 0.83 for heifers and 0.82 for cows. In Germany, few females are currently genotyped. Therefore, the NRR of risk mating, meaning that the embryo's sire and maternal grandsire both carry a specific characteristic, were compared with NRR of nonrisk mating where both were noncarriers. The NRR of risk mating were compared with NRR of nonrisk mating with a $t$-test to investigate whether a significant difference between mating groups exists. The economic value of embryo loss was estimated as costs for an additional insemination and costs for increased calving interval.

The economic value of polled was estimated to be $€ 7$ per calf (€5 for labor and $€ 2$ for drugs). The social policy value and the current higher marketing price of a polled calf were not considered. The economic value of $€ 7$ per polled calf is in agreement with Widmar et al. (2013), who compared different dehorning scenarios. The expected costs of dehorning varied from $\$ 5.84$ to $\$ 22.89$ with a mean of $\$ 11.79$.

No extra costs for direct gene tests were considered because causal mutation can be easily added to customized chips used in routine genomic evaluation.

\section{Genetic Index}

Table 2 illustrates the average economic effect of a genetic characteristic in a given population (Falconer and MacKay, 1996). In general, the effect on the population depends on the genotype of the animal, the allele frequency (p and q) and the economic value $(\alpha)$. The genetic index (GI) of an animal is the sum of all considered characteristics:

$$
\mathrm{GI}=\sum_{\mathrm{k}=1}^{\mathrm{n}} \mathrm{A} \mathrm{V}_{\mathrm{k}}
$$

where AVk is the average economic effect of the genetic characteristic $\mathrm{k}$, and $\mathrm{n}$ is the total number of considered genetic characteristics.

The influence of a genetic index on production and functional breeding values and allele frequencies were studied by a simulation of a genomic breeding program. Additionally, the genetic index was applied to real data by calculating the genetic index for each genotyped animal.

\section{Simulation of a Genomic Breeding Program}

The genomic breeding program was simulated as reported by Täubert et al. (2011) and Segelke et al. (2013). The breeding program had a size of 250,000 dairy cows under milk recording, from which the best $1 \%$ were selected as bull dams based on their breeding values. From these dams, 1,250 male selection candidates were born, 500 bull calves were genotyped out of the 1,250 candidates. Based on their genomic breeding values, 29 of these 500 bull calves were selected and used as service sires as soon as they produced semen. One bull was selected to go on to become a second crop service sire. To realize as much genetic gain as possible, $95 \%$ of sire of sons are young genomically tested bulls. Only $5 \%$ of all sires of sons are also daughter proven. Cow sires are $94 \%$ genomically proven and $6 \%$ daughter-proven bulls.

The breeding values of the cow base population had a mean of 100 and a standard deviation of 20 . The reliabilities of the estimated breeding values were $50 \%$. Artificial insemination bulls are superior compared with the cows; therefore, 500 bulls were simulated in the first generation with a mean of 130 and a standard deviation of 5 . The best 30 animals were selected as sires. The reliability for the estimated breeding values was $67 \%$ for the genomic bulls and $99 \%$ for the daughter-proven bulls. The breeding value of an individual animal was simulated as sum of the true breeding value, a Mendelian sampling part, and a nonheritable residual effect. No mating of closely related animals occurred. A total of 10 generations were simulated and each scenario was repeated 100 times. Starting allele frequencies for the

Table 2. Effect of a genetic characteristic on a given population (Falconer and MacKay, 1996)

\begin{tabular}{|c|c|}
\hline $\begin{array}{l}\text { Genotype of } \\
\text { animal }\end{array}$ & $\begin{array}{l}\text { Average economic effect } \\
\text { in a given population }{ }^{1}\end{array}$ \\
\hline $\begin{array}{l}\mathrm{AA} \\
\mathrm{AB} \\
\mathrm{BB}\end{array}$ & $\begin{array}{c}2 q \alpha \\
(q-p) \alpha \\
-2 p \alpha\end{array}$ \\
\hline
\end{tabular}


base population were the average allele frequency of the animals born between 2012 and 2014 (Table 1).

Two different breeding scenarios for the females were analyzed:

- Scenario A: All animals were selected using their breeding values. The genetic index was not considered.

- Scenario I: Animals on the dam path were ranked and selected due to the genetic index. The breeding value was not considered.

In both scenarios, the AI bulls were selected due to the estimated breeding values to maintain the genetic gain for the production and functional traits.

\section{RESULTS AND DISCUSSION}

\section{Validation of the Missing Homozygosity Approach}

The comparison between the genotypes of HH1, HH3, and HH4 from the custom SNP array and the genotypes derived from the missing homozygotes approach is shown in Table 3. Two genotyped embryos were homozygous BB for HH3. In total, 7,032 animals were compared and a high agreement between the SNP chip-based and haplotype-based results were found (genotype error rate below $0.3 \%$ ). For all 3 defects, the call rate was $99.8 \%$. For HH4 no unequal genotypes were recorded, meaning that the $2 \mathrm{SNP}$ which relate to the same position show the same results. For HH1 $0.09 \%$ of all animals had unequal genotypes, although the identical position was analyzed.

\section{Carrier Frequencies of the Considered Traits}

The development of the haplotype based carrier frequencies over the years of birth for the 6 consid- ered traits is illustrated in Figure 1. No evidence for a selection against the recessive haplotypes was found. Carrier frequencies of $\mathrm{HH} 1, \mathrm{HH} 2$, and $\mathrm{HH} 4$ are actually decreasing. In contrast, carrier frequencies of $\mathrm{HH} 3$ and HH5 are still increasing because popular sires such as Picston Shottle and O-Bee Manfred Justice-ET, with a high influence on the actual German Holstein population, are carriers of these haplotypes. The famous sire Jocko Besn carries HH4 and has a lot of popular sons, resulting in an increase of the carrier frequency for the birth years 2005 to 2007. The sons of Jocko Besn were famous cow sires; consequently, the allele frequency of HH4 decreases because mainly male animals were genotyped at this time. The polled phenotype is increasing since 2010 because of the social policy force to avoid dehorning. As reported by Segelke et al. (2013), the gain mainly based on the excessive use of the sire Aggravation Lawn Boy P-Red, which has resulted in a high inbreeding level in polled animals. Minor allele frequencies of birth years 1990 to 2009 were mainly based on genotyped bulls representing the genomic reference population. However, due to an increase in numbers of genotyped cows including genotyping complete herds in the near future, the genotype pool from genomic evaluation can be used as a monitoring system for genetic characteristics. Figure 1 shows that strategies to reduce and manage allele frequencies especially for $\mathrm{HH} 3$ and HH5 are needed.

\section{Effect of Missing Homozygote Haplotypes on Fertility and Economic Values}

To determine the time of embryo loss for each recessively inherited disorder of fertility, NRR56 and NRR90 for heifers and cows were analyzed. Based on this timing, the approximate economic value per recessive defect was estimated (Table 4). For HH1 a decrease of NRR56 in heifers and cows was observed for risk mat-

Table 3. Comparison between carrier states derived from custom SNP array and indirect genotype derived from haplotypes

\begin{tabular}{|c|c|c|c|c|c|c|c|}
\hline \multirow[b]{2}{*}{ Item } & \multirow[b]{2}{*}{ Haplotype } & \multirow[b]{2}{*}{ Carrier state } & \multicolumn{5}{|c|}{ Chip based ${ }^{1}$} \\
\hline & & & AA & $\mathrm{AB}$ & $\mathrm{BB}$ & No call & Error call \\
\hline \multirow{6}{*}{ Haplotype based } & & $\mathrm{AB}$ & 0 & 109 & 0 & 5 & 4 \\
\hline & & BB & 0 & 0 & 0 & 0 & 0 \\
\hline & HH3 & AA & 6,673 & 23 & 0 & 6 & 2 \\
\hline & & $\mathrm{AB}$ & 0 & 323 & 0 & 1 & 2 \\
\hline & & $\mathrm{AB}$ & 2 & 339 & 0 & 2 & 0 \\
\hline & & BB & 0 & 0 & 0 & 0 & 0 \\
\hline
\end{tabular}

${ }^{1} \mathrm{AA}=$ homozygote noncarrier; $\mathrm{AB}=$ heterozygote carrier; $\mathrm{BB}=$ homozygote carrier; No call $=1$ or 2 SNP (identical genome position) without a result; Error call = results of the both SNP (identical genome position) were unequal. 


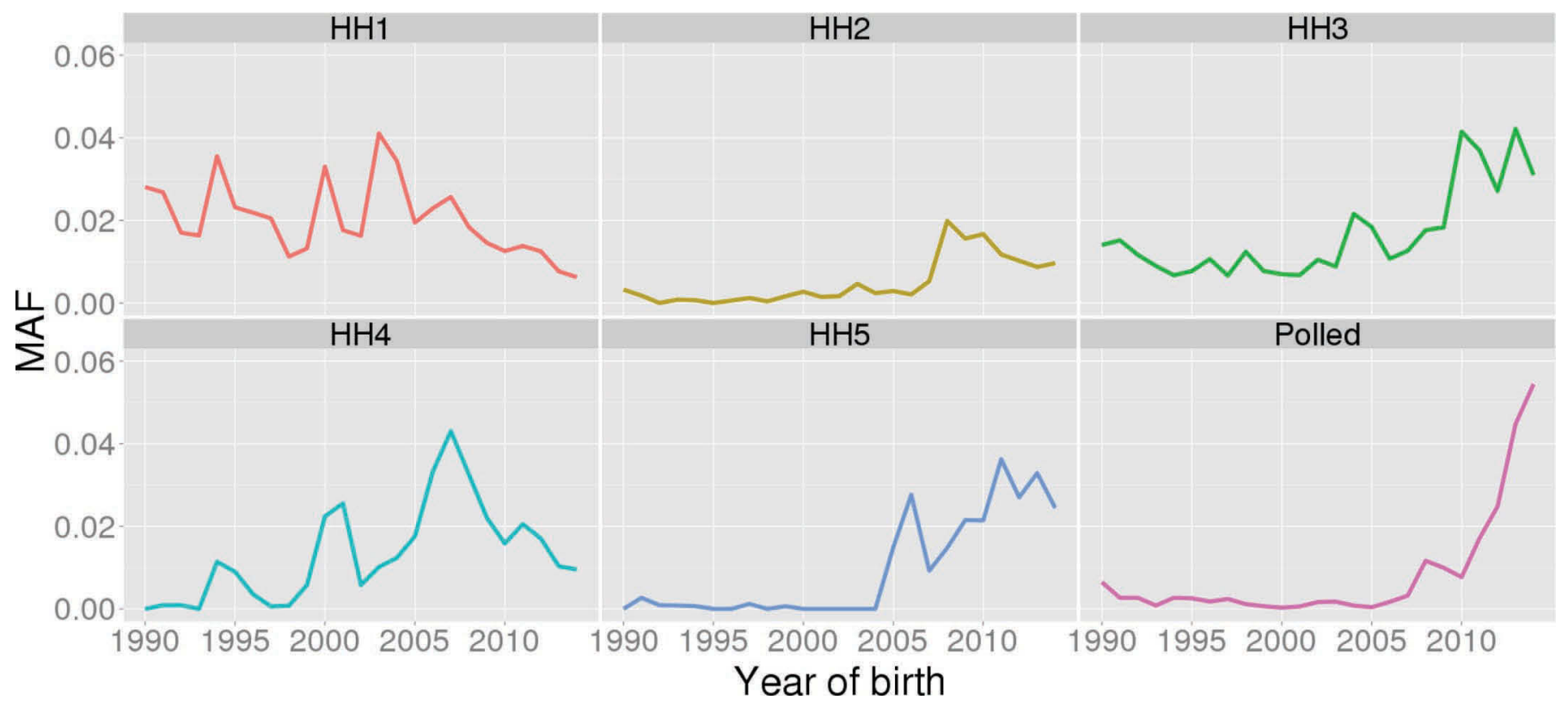

Figure 1. Minor allele frequencies (MAF) of the analyzed recessive fertility defects and polled. Color version available online.

ing in comparison with nonrisk mating. Additionally, a higher negative effect on NRR90 was found in contrast to NRR56, indicating that the embryo dies before d 90 of gestation. The estimation of the economic value per lost embryo was based on the averaged day of the embryo's death. The average date of death at $\mathrm{d} 63$ includes a 63-d extended calving interval. Assuming marginal costs of $€ 1.3$ per day of extended calving interval and $€ 15$ per insemination results in a total economic value of $€ 97$ per lost embryo for HH1. The allele frequency of HH2 was very low during the last years (Figure 1), preventing the occurrence of risk mating. No significant effect was found in this data set. Similar to HH1, it was assumed that the embryo dies on average on $\mathrm{d}$ 63 meaning an economic loss of $€ 97$ occurs. For HH3, HH4, and HH5, a main effect on NRR56 was observed, implying the death of the embryo before $d 56$. It was assumed that on average the embryo dies on $d 42$ (2 fertility cycles), yielding in an economic value per lost embryo of $€ 70$.

The estimated effects of HH1 on NRR are in agreement with VanRaden et al. (2011b). They reported an effect of $-1.1 \%$ on NRR60 and $-1.6 \%$ on NRR100. The authors concluded that the embryo dies throughout the gestation. Fritz et al. (2013) found a higher effect of HH1 on overall heifer and cow calving rate. Nonsignificant results of $\mathrm{HH} 2$ are in agreement with Fritz et al. (2013), who also were not able to significantly ensure the effect on NRR because of the low number of risk mating. VanRaden et al. (2011b) reported an effect on NRR60 of $-1.7 \%$ and $-3.0 \%$ for NRR100, supporting our hypothesis that the embryo dies between d 60 and 100. However, VanRaden et al. (2011b) concluded that for HH2 the embryo loss is mainly before $60 \mathrm{~d}$. The re-

Table 4. Effect of recessive defects on fertility phenotypes ${ }^{1}$

\begin{tabular}{|c|c|c|c|c|c|}
\hline \multirow[b]{2}{*}{ Characteristic } & \multicolumn{2}{|c|}{ Heifer } & \multicolumn{2}{|c|}{ Cow } & \multirow{2}{*}{$\begin{array}{l}\text { Economic } \\
\text { value per } \\
\text { embryo }(€)\end{array}$} \\
\hline & $\Delta \underset{(\%)}{\operatorname{NRR} 56}$ & $\Delta \underset{(\%)}{\operatorname{NRR90}}$ & $\Delta \underset{(\%)}{\operatorname{NRR56}}$ & $\Delta \underset{(\%)}{\operatorname{NRR90}}$ & \\
\hline HH1 & $-2.0 \pm 0.5$ & $-3.2 \pm 0.5$ & $-2.5 \pm 0.5$ & $-3.5 \pm 0.5$ & -97 \\
\hline $\mathrm{HH} 2$ & $0.1 \pm 1.6^{\mathrm{NS}}$ & $0.1 \pm 1.6^{\mathrm{NS}}$ & $0.2 \pm 1.4^{\mathrm{NS}}$ & $0.8 \pm 1.4^{\mathrm{NS}}$ & -97 \\
\hline HH3 & $-4.1 \pm 0.8$ & $-4.3 \pm 0.8$ & $-5.0 \pm 0.7$ & $-5.6 \pm 0.7$ & -70 \\
\hline $\mathrm{HH} 4$ & $-2.4 \pm 0.4$ & $-2.8 \pm 0.4$ & $-1.6 \pm 0.5$ & $-2.1 \pm 0.4$ & -70 \\
\hline HH5 & $-5.4 \pm 1.5$ & $-5.4 \pm 1.5$ & $-8.9 \pm 1.3$ & $-9.2 \pm 1.3$ & -70 \\
\hline
\end{tabular}

${ }^{1} \mathrm{NRR}=$ nonreturn rate; $\Delta=\mathrm{NRR}$ nonrisk mating $-\mathrm{NRR}$ risk mating. NS $(P>0.05)$. 


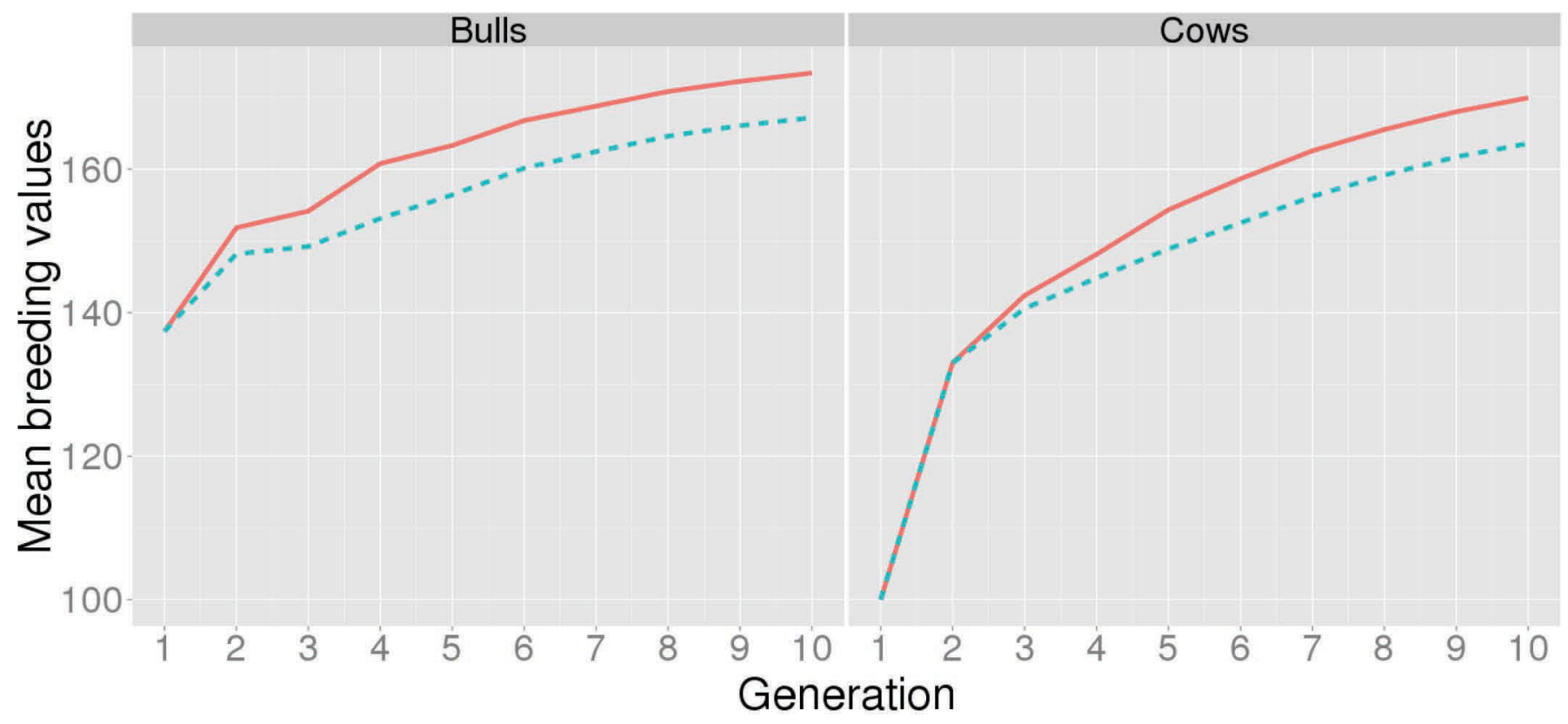

Figure 2. Development of the breeding values over 10 generations. In scenario A (solid line), all animals were selected using their breeding values. In scenario I (dotted line), animals on the dam path were ranked and selected due to the genetic index. Color version available online.

sults of HH3 and early embryonic loss are in agreement with VanRaden et al. (2011b) and Fritz et al. (2013). The estimated effects on fertility phenotypes mainly depend on the allele frequency in the population. The estimated effects between countries differ because of differences in the use of bull sires between the countries.

The estimated economic values in this study are very similar because currently mainly disorders causing abortion are known (i.e., are associated with fertility). Genetic disorders occur across breeds and across species (Nicholas and Hobbs, 2014). Recently many genetic disorders were identified in the Simmental breed (Jung et al., 2014; Pausch et al., 2015). These disorders have diverse allele frequencies, and time points of embryo or calf loss lead to different economic values. Egger-Danner et al. (2014) showed that losses for Fleckvieh-haplotype 2, dwarfism, and zinc-deficiency-like syndrome have an economic value of $€ 350$ because the calf is not marketable. Considering veterinary costs, an even higher economic loss is realistic. Costs for arachnomelia are approximately $€ 700$ because additionally a damage of the cow might occur (Egger-Danner et al., 2014). The authors expect a $7 \%$ loss in annual monetary genetic gain and a $9 \%$ reduction in discounted profit when all male carriers of the 6 considered defects are excluded from mating. Ongoing identification of genetic defects leads to more difficulties in the exclusion of all carriers from mating. Van Eenennaam and Kinghorn (2014) showed that decrease of genetic gain depends on number of lethal loci and allele frequencies. Selection against a small number of defects with intermediate allele frequencies reduced genetic gain to $92.5 \%$. However, if 100 loci were modeled, the genetic gain decreased to $86 \%$.

\section{Genetic Index}

The change in breeding values over 10 generations for bulls and dams are shown in Figure 2. An increase of the breeding values is expected in the next generations. Changing the mating strategy of the female side from assortative mating to selection on genetic index (scenario I) results in a loss of genetic progress compared with scenario A. Lower selection intensity is the reason for lower genetic response. Figure 3 shows the development of the genetic index over 10 generations for both scenarios. Selecting the females due to scenario I instead of selecting them due to the breeding values (scenario A) results in a high increase of the genetic index in both sexes.

The development of the allele frequencies for the 6 analyzed traits is illustrated in Figure 4. Recessive fertility defects are slightly decreasing over time because homozygote embryos do not survive. The polled genotype slightly increased in scenario A because of the dominant inheritance. In scenario I, a high increase of the polled allele frequency occurs because the positive economic value results in an additional selection for polled. In comparison, including the fertility disorders 


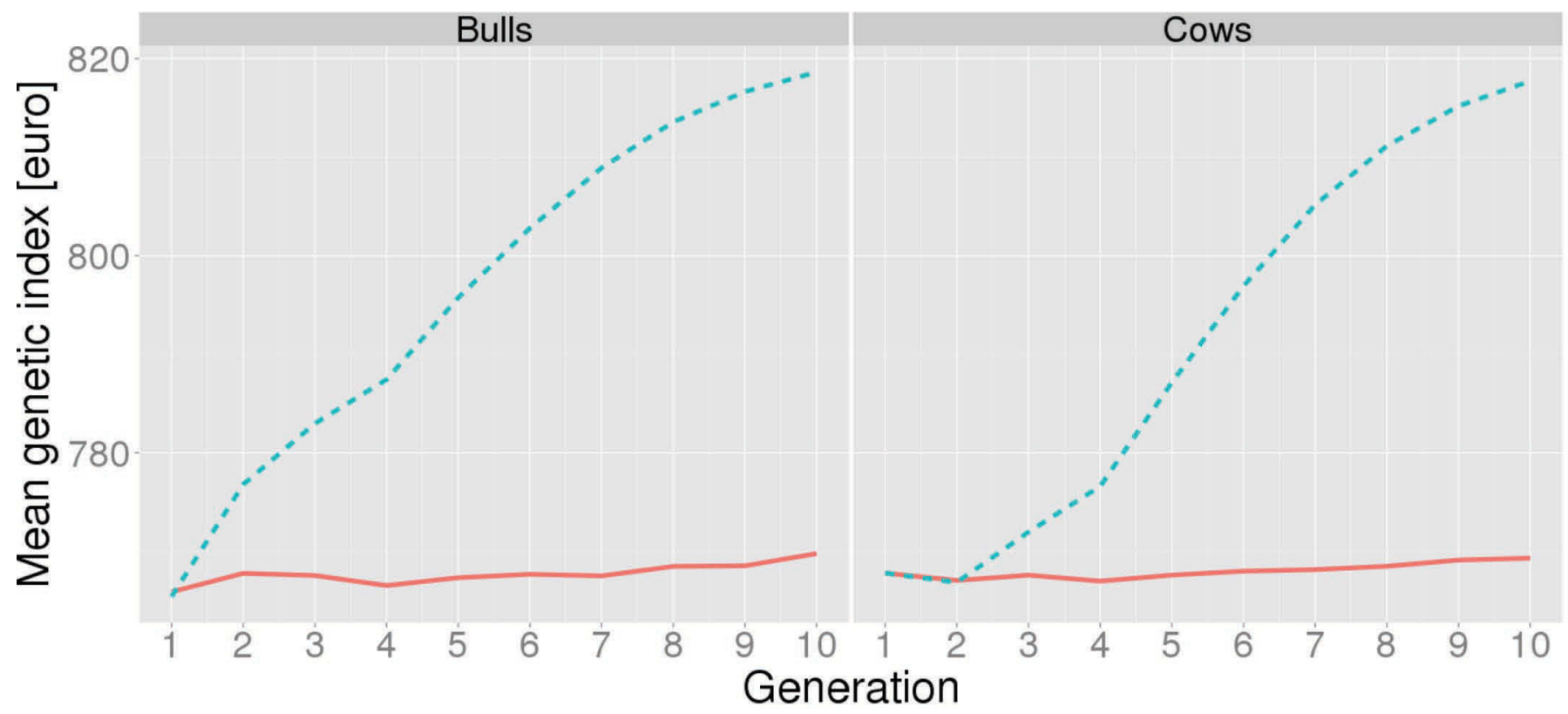

Figure 3. Development of the genetic index over 10 generations. In scenario A (solid line), all animals were selected using their breeding values. In scenario I (dotted line), animals on the dam path were ranked and selected due to the genetic index. Color version available online.

with a negative economic value in an index will provide more selection pressure than relying on selection against their small effects on overall fertility. In conclusion, selection for a genetic index considering the economic value is an efficient method to control the allele frequen- cies by reducing undesirable alleles and simultaneously increasing economically beneficial traits. However, our assumption was that bulls were only ranked according their breeding values and females were primarily ranked on their genetic index. In practice, farmers and
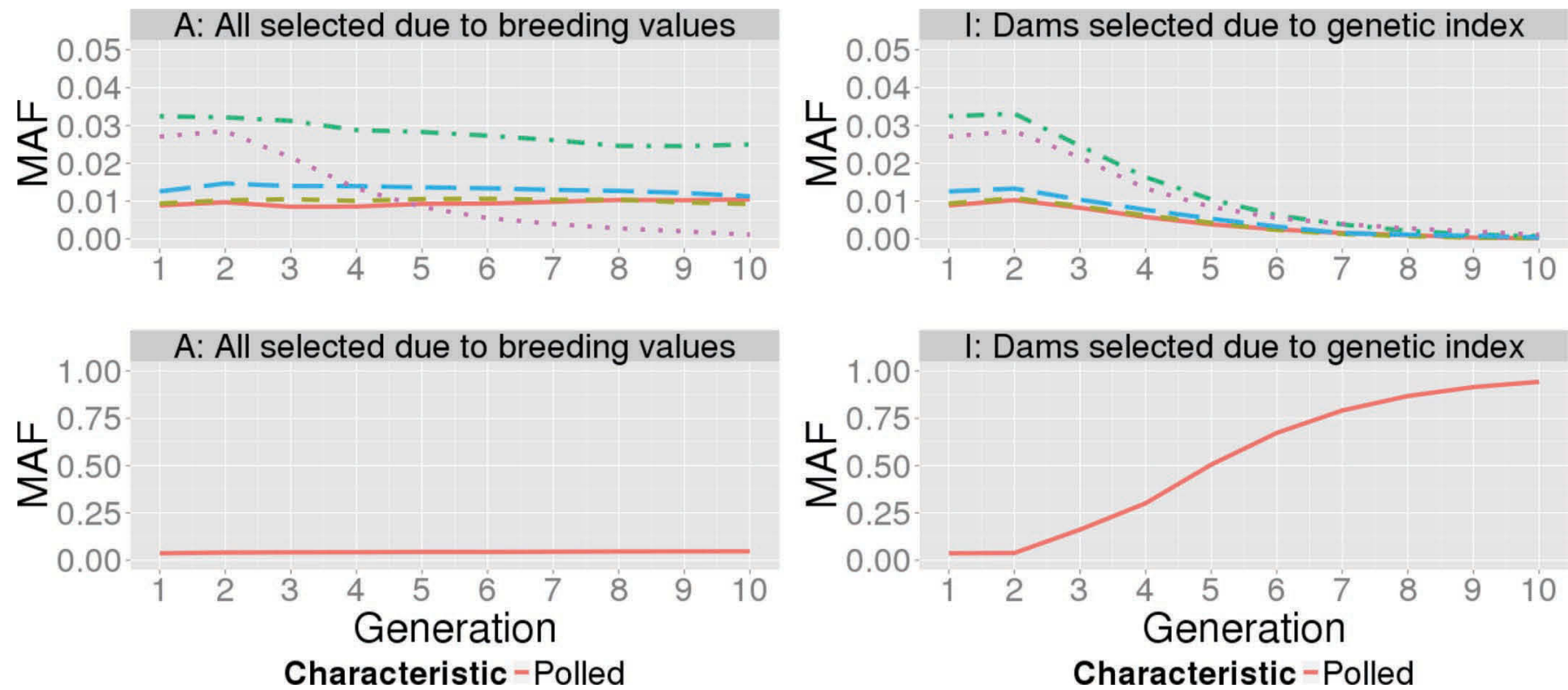

Figure 4. Development of the mean allele frequencies (MAF) of genotypes HH1 to HH5 over 10 generations. In scenario A (solid line), all animals were selected using their breeding values. In scenario I (dotted line), animals on the dam path were ranked and selected due to the genetic index. HH1 = solid line; HH2 = short dash line; HH3 = long dash line; HH4 = dash dot line; HH5 = dotted line). Color version available online. 


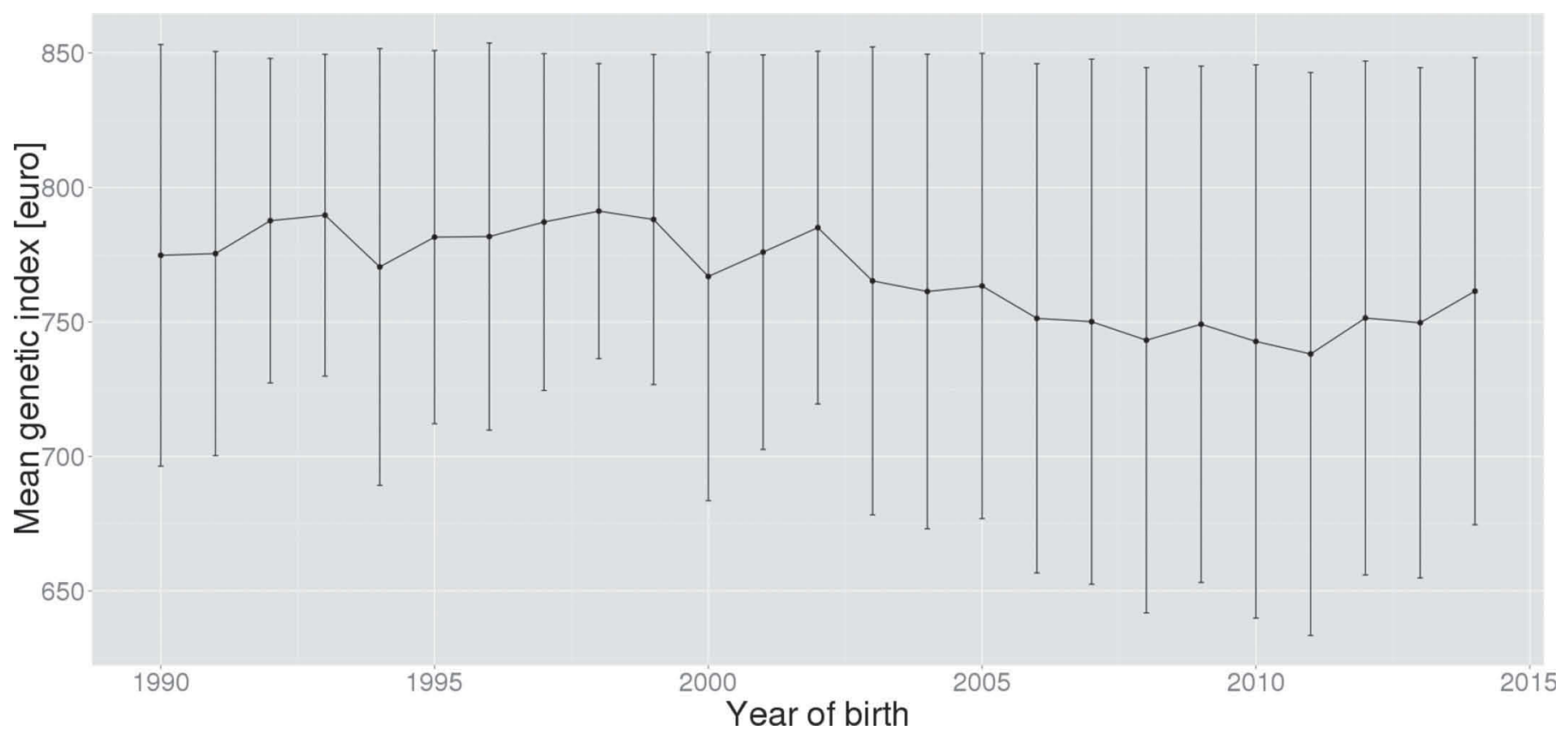

Figure 5. Mean and standard deviation of the genetic index applied to genotyped animals for the birth years 1990 to 2014 .

AI organizations will select and mate animals using a combination of breeding values in addition to a genetic index. Before applying our method, further research is needed to investigate the consequence of selecting the animals based on a combination of breeding values and genetic index.

\section{Genetic Index Applied to Real Data}

The concept of an index for genetic characteristics as the sum of the specific attributes with respect to their allele frequencies in the population and their economic values (Table 1) was applied to the genotype data pool from routine genomic evaluation. For all these individuals, an animal-specific genetic index was calculated. A decrease of the genetic trend of $€ 30$ on average can be found for the birth years 1990 to 2010 (Figure 5). This implies that the overall number of genetic defects in the population increased over time. This can be explained by high frequencies of HH1 from 1990-2006. Afterward, frequencies of $\mathrm{HH} 3$ and $\mathrm{HH} 5$ with a lower economic value compared with HH1 increased. Since 2010, the polled frequency is strongly increasing, which raises the genetic index.

Analyzing the total number of known recessive defects each animal carries showed that $83.21 \%$ of all genotyped animals were free from all considered genetic defects; $15.68 \%$ carried 1 genetic defect, $1.07 \%$ carried 2 genetic defects, $0.04 \%$ had 3 defects, and one animal carried 4 fertility defects.
We also investigated the relationship between the genetic index and genomic enhanced fertility breeding values. No significant association between fertility breeding values and the genetic index was noticed because most of the animals do not carry any currently known genetic defect. No associations between number of defects and fertility breeding values were found. This implies that breeding values slightly consider known recessive defects because of small allele substitutions effects. Additional effects of the fertility disorders on fertility breeding values depend on the allele frequencies in the population. Intermediate allele frequencies would lead to significant embryo loss. Most of the considered fertility defects had low and fluctuating minor allele frequencies. Taking the genetic defects into account avoids carrier mating. This eliminates the risk of homozygous affected embryos and allows for a quick decrease of the allele frequencies. Mating only by fertility breeding values cannot limit the increase of the allele frequencies (Figure 1).

\section{Future Aspects}

Routine genotyping of large proportion of the population allows discovering, managing, and monitoring genetic disorders and economical beneficial genetic characteristics. However, even after applying next generation sequencing technologies, for some defects the causal mutation is still unknown (McClure et al., 2014). For defects where the mutation is known, ad- 
ditional research is needed to understand the biology of the mutation. Analyzing data from embryo transfer stations might be an opportunity to more precisely determine the time of embryonic loss, compared with the indirect use of NRR data sets. Additionally, more research is needed to determine whether the genetic effects of the recessive alleles are already to some extent included in the fertility breeding values and how the genetic index can be integrated into total merit indices. Genomic mating plans are needed for computer-based accounting for all genetic characteristic according to their economic values.

\section{CONCLUSIONS}

Ongoing research will detect a number of new genetic disorders. Consequently, demand is clear to develop a method combining selection against recessive defects with selection for economically beneficial traits. Our proposed method is a genetic index that combines different genetic characteristics with different economic values and allele frequencies. For breeding decisions, the index should be used for the female path and bulls should be selected due to breeding values of production and functional traits to maintain the genetic gain. Mating recommendations should be calculated using mating programs taking all genetic characteristics of mating partners into account. However, further investigation is needed to determine the correct time of embryo loss and the economic value.

\section{ACKNOWLEDGMENTS}

The German national organization Förderverein Bioökonomieforschung e.V. (FBF, Bonn, Germany) is thanked for financial support.

\section{REFERENCES}

Adams, H. A., T. Sonstegard, P. M. VanRaden, D. J. Null, C. Van Tassell, and H. Lewin. 2012. Identification of a nonsense mutation in APAF1 that is causal for a decrease in reproductive efficiency in dairy cattle. Abstr. P0555 in Proc. Plant Anim. Genome XX Conf.

Agerholm, J. S., C. Bendixen, O. Andersen, and J. Arnbjerg. 2001 Complex vertebral malformation in Holstein calves. J. Vet. Diagn. Invest. 13:283-289.

Agerholm, J. S., F. McEvoy, and J. Arnbjerg. 2006. Brachyspina syndrome in a Holstein calf. J. Vet. Diagn. Invest. 18:418-422.

Bendixen, C., S. Soren, J. Helle, P. Frank, P. A. Aasberg, H. Lars-Erik, H. Per, H. Anette, T. Bo, J. Mette, N. B. Vivi Hunnicke, and J. M. Ssergij. 2014. Genetic test for the identification of carriers of complex vertebral malformations in cattle. US20140242585. Accessed Apr. 14, 2015. https://patentscope.wipo.int/search/en/detail.jsf? docId $=$ US107213162\&redirectedID $=$ true.

Browning, S. R., and B. L. Browning. 2007. Rapid and accurate haplotype phasing and missing data inference for whole genome association studies by use of localized haplotype clustering. Am. J. Hum. Genet. 81:1084-1097.
Charlier, C., J. S. Agerholm, W. Coppieters, P. Karlskov-Mortensen, W. Li, G. de Jong, C. Fasquelle, L. Karim, S. Cirera, N. Cambisano, N. Ahariz, E. Mullaart, M. Georges, and M. Fredholm. 2012. A deletion in the bovine FANCI gene compromises fertility by causing fetal death and brachyspina. PLoS ONE 7:e43085.

Cooper, T. A., G. R. Wiggans, D. J. Null, and J. L. Hutchison. 2013 Genomic evaluation and identification of a haplotype affecting fertility for Ayrshire dairy cattle. J. Dairy Sci. 96(E-Suppl. 1):74. (Abstr.)

Daetwyler, H. D., A. Capitan, H. Pausch, P. Stothard, R. van Binsbergen, R. F. Brøndum, X. Liao, A. Djari, S. C. Rodriguez, C. Grohs, D. Esquerré, O. Bouchez, M.-N. Rossignol, C. Klopp, D. Richa, S. Fritz, A. Eggen, P. J. Bowman, D. Coote, A. J. Chamberlain, C. Anderson, C. P. Van Tassell, I. Hulsegge, M. E. Goddard, B. Guldbrandtsen, M. S. Lund, R. F. Veerkamp, D. A. Boichard, R. Fries, and B. J. Hayes. 2014. Whole-genome sequencing of 234 bulls facilitates mapping of monogenic and complex traits in cattle. Nat. Genet. 46:858-865.

Egger-Danner, C., H. Schwarzenbacher, C. Fuerst, and A. Willam. 2014. Analysis of breeding strategies against genetic disorders in Austrian Fleckvieh cattle. Article No. 400 in 10th World Congress on Genetics Applied to Livestock Production, Vancouver, Canada. https://asas.confex.com/asas/WCGALP14/webprogram/start. html.

Falconer, D. S., and F. C. MacKay. 1996. Introduction to Quantitative Genetics. 4th ed. John Wiley \& Sons, New York, NY.

Fritz, S., A. Capitan, A. Djari, S. C. Rodrigue, A. Barbat, A. Baur, C. Grohs, B. Weiss, M. Boussaha, D. Esquerré, C. Klopp, D. Rocha, and D. Boichard. 2013. Detection of haplotypes associated with prenatal death in dairy cattle and identification of deleterious mutations in GART, SHBG and SLC37A2. PLoS ONE 8:e65550.

Georges, M., W. Coppieters, C. Charlier, J. S. Agerholm, and M. Fredholm. 2010. A genetic test for Brachyspina and fertility in cattle. Patent application WO2010012690. Accessed Apr. 12, 2015. http://www.wipo.int/patentscope/search/en/WO2010012690.

Jansen, S., B. Aigner, H. Pausch, M. Wysocki, S. Eck, A. Benet-Pagès, E. Graf, T. Wieland, T. M. Strom, T. Meitinger, and R. Fries. 2013. Assessment of the genomic variation in a cattle population by re-sequencing of key animals at low to medium coverage. BMC Genomics 14:446.

Jung, S., H. Pausch, M. C. Langenmayer, H. Schwarzenbacher, M. Majzoub-Altweck, N. S. Gollnick, and R. Fries. 2014. A nonsense mutation in PLD4 is associated with a zinc deficiency-like syndrome in Fleckvieh cattle. BMC Genomics 15:623.

McClure, M. C., D. Bickhart, D. Null, P. VanRaden, L. Xu, G. Wiggans, G. Liu, S. Schroeder, J. Glasscock, J. Armstrong, J. B. Cole, C. P. Van Tassell, and T. S. Sonstegard. 2014. Bovine exome sequence analysis and targeted SNP genotyping of recessive fertility defects BH1, HH2, and HH3 reveal causative mutation in SMC2 for HH3. PLoS ONE 9:e92769.

Medugorac, I., D. Seichter, A. Graf, I. Russ, H. Blum, K. H. Göpel, S. Rothammer, M. Förster, and S. Krebs. 2012. Bovine polledness-An autosomal dominant trait with allelic heterogeneity. PLoS ONE 7:e39477.

Nicholas, F., and M. Hobbs. 2014. Mutation discovery for Mendelian traits in nonlaboratory animals: A review of achievements up to 2012. Anim. Genet. 45:157-170.

Pausch, H., H. Schwarzenbacher, J. Burgstaller, K. Flisikowski, C. Wurmser, S. Jansen, S. Jung, A. Schnieke, T. Wittek, and R. Fries. 2015. Homozygous haplotype deficiency reveals deleterious mutations compromising reproductive and rearing success in cattle. BMC Genomics 16:312.

Rothammer, S., A. Capitan, E. Mullaart, D. Seichter, I. Russ, and I. Medugorac. 2014. The $80-\mathrm{kb}$ DNA duplication on BTA1 is the only remaining candidate mutation for the polled phenotype of Friesian origin. Genet. Sel. Evol. 46:44.

Sahana, G., U. S. Nielsen, G. P. Aamand, M. S. Lund, and B. Guldbrandtsen. 2013. Novel harmful recessive haplotypes identified for fertility traits in Nordic Holstein cattle. PLoS ONE 8:e82909. 
Segelke, D., H. Täubert, F. Reinhardt, and G. Thaller. 2013. Chancen und Grenzen der Hornloszucht für die Rasse Deutsche Holstein. Zuchtungskunde 85:4.

Shanks, R. D., D. B. Dombrowski, G. W. Harpestad, and J. L. Robinson. 1984. Inheritance of UMP synthase in dairy cattle. J. Hered. 75:337-340.

Shuster, D. E., M. E. Kehrli Jr., M. R. Ackermann, and R. O. Gilbert. 1992. Identification and prevalence of a genetic defect that causes leukocyte adhesion deficiency in Holstein cattle. Proc. Natl. Acad. Sci. USA 89:9225-9229.

Täubert, H., S. Rensing, and F. Reinhardt. 2011. Comparing conventional and genomic breeding programs with ZPLAN+. Interbull Bull. 44:162-168.

Van Eenennaam, A. L., and B. P. Kinghorn. 2014. Use of mate selection software to manage lethal recessive conditions in livestock populations. Article No. 408 in Proc. 10th World Congress on Genetics Applied to Livestock Production, Vancouver, Canada. https://asas.confex.com/asas/WCGALP14/webprogram/start. html.

VanRaden, P. M., K. M. Olson, D. J. Null, and J. L. Hutchison. 2011a. Harmful recessive effects on fertility detected by absence of homozygous haplotypes. J. Dairy Sci. 94:6153-6161.
VanRaden, P. M., K. M. Olson, D. J. Null, and J. L. Hutchison. 2011b. Reporting of haplotypes with recessive effects on fertility. Presentations \& Papers from the 2011 Interbull Open Meeting. Accessed Apr. 4, 2015. https://journal.interbull.org/index.php/ib/article/ view/1202/1287.

VanRaden P. M., C. Sun, T. A Cooper, D. J. Null, and J. B. Cole. 2014. Keynote presentation III: Genotypes are useful for more than genomic evaluation. Pages 19-23 in Proc. 39th Int. Commun. Anim. Recording Sess., Berlin, Germany.

VIT. 2015. Estimation of breeding values for milk production traits, somatic cell score, conformation, productive life and reproduction traits in German dairy cattle. Accessed Apr. 4, 2015. http://www.vit.de/fileadmin/user_upload/vit-fuers-rind/ zuchtwertschaetzung/milchrinder-zws-online/Zws_Bes_eng.pdf.

Widmar, N. J. O., M. M. Schutz, and J. B. Cole. 2013. Breeding for polled dairy cows versus dehorning: Preliminary cost assessments and discussion. J. Dairy Sci. 96(E-Suppl. 1):602. (Abstr.) 